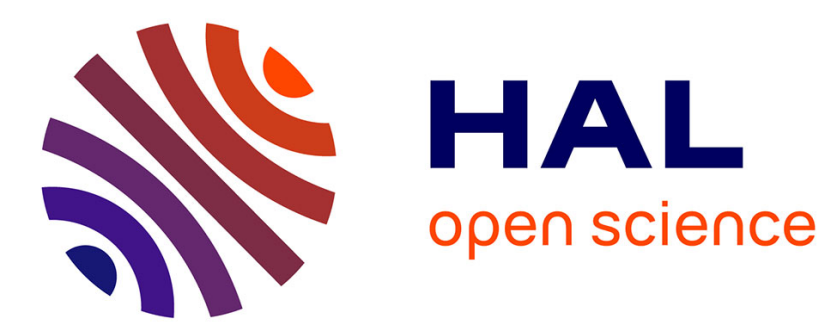

\title{
A fully automatized processing chain for high-resolution multispectral image acquisition of crop parcels by UAV
}

Gilles Rabatel, S. Labbé

\section{To cite this version:}

Gilles Rabatel, S. Labbé. A fully automatized processing chain for high-resolution multispectral image acquisition of crop parcels by UAV. 10th European Conference on Precision Agriculture (ECPA 2015), Jul 2015, Beit Dagan, Israel. pp.135-141, 10.3920/978-90-8686-814-8_16 . hal-01342117

\section{HAL Id: hal-01342117 \\ https://hal.science/hal-01342117}

Submitted on 5 Jul 2016

HAL is a multi-disciplinary open access archive for the deposit and dissemination of scientific research documents, whether they are published or not. The documents may come from teaching and research institutions in France or abroad, or from public or private research centers.
L'archive ouverte pluridisciplinaire HAL, est destinée au dépôt et à la diffusion de documents scientifiques de niveau recherche, publiés ou non, émanant des établissements d'enseignement et de recherche français ou étrangers, des laboratoires publics ou privés. 


\title{
A fully automatized processing chain for high-resolution multispectral image acquisition of crop parcels by UAV
}

\author{
Rabatel G. ${ }^{1}$, Labbé S. ${ }^{2}$ \\ ${ }^{1}$ Irstea, 361 rue J-F Breton, BP 5095, 34196 Montpellier Cedex 5, France \\ ${ }^{2}$ Maison de la Télédétection, 500 rue J-F Breton 34093 Montpellier Cedex 5, France \\ gilles.rabatel@irstea.fr
}

\begin{abstract}
In the context of the RHEA European project addressing site-specific weeding on arable crops, a complete aerial image acquisition chain has been developed, providing a georeferenced multispectral image of a total parcel with $1 \mathrm{~cm}$ spatial resolution. Robust automatic ground control point detection and near infrared - visible sensor image registration modules have been implemented and associated with the MicMac photogrammetric software suite to provide a fully automatized mosaicking procedure. This procedure has been successfully applied on various maize and wheat crops for weed patch detection, but could be extended to other precision agriculture applications, especially those requiring a very high spatial resolution.
\end{abstract}

Keywords Near infrared image, mosaicking, ground control point

\section{Introduction}

Unmanned aerial vehicles (UAV) have emerged as an alternative to aircraft and satellite remote sensing for agricultural monitoring, especially in the context of precision agriculture (Zhang \& Kovacs. 2012). Indeed, UAV provide significant advantages: short revisit time due to their great flexibility in flight scheduling, image usability in cloudy conditions, and very high spatial resolution thanks to low altitude flight capabilities.

As a counterpart, UAV operation has important limitations in terms of flight duration, positioning accuracy, available payload and power source for imaging devices. As a consequence, most embedded cameras are limited to RGB image acquisition (Shahbazi et al, 2014). Although some commercial multispectral devices are available (e.g. Tetracam, Chatsworth, CA, USA), they usually do not allow a spatial resolution better than $10 \mathrm{~cm}$ (Xiang, H., Tian, L., 2011, García-Ruiz et al, 2013).

In this context, the European FP7 project RHEA (2010-2014), devoted to site specific weeding management in arable crops, had to face a cutting-edge challenge. One of the tasks to achieve was to perform an aerial inspection of the targeted crop parcels in order to locate weed patches for further weeding operation. Due to the poor feasibility of spectral approaches for weed detection (Slaughter et al, 2008), an approach based on crop row detection has been chosen (Gée et al, 2008). However, in the case of winter 
wheat where the inter-row is about $15 \mathrm{~cm}$, crop row detection requires a centimetric spatial resolution (Torres-Sánchez et al, 2014). Moreover, a multispectral image including a near infrared band (NIR) is required for robust discrimination between vegetation and soil in any lighting conditions (Peña et al, 2014).

A UAV vision system made up of two compact still cameras has been designed to meet both these requirements, i.e. high spatial resolution and multispectral acquisition. It provides color and NIR images with a spatial resolution of $1 \mathrm{~cm}$ at $60 \mathrm{~m}$ altitude. As a counterpart, numerous image acquisitions are necessary to cover the whole parcel.

The software processing chain which consists in merging images is known as mosaicking. Many commercial or open source mosaicking software solutions are now available. However, they usually lead to a loss of spatial accuracy in the resulting image (Zhang \& Kovacs. 2012). In the present case, in order to preserve as much as possible the initial image accuracy, open source software MicMac (MicMac, IGN, France) has been chosen. This software offers a high degree of flexibility in terms of computation algorithms, distortion model definition, etc. In counterpart, it needs the management of many input parameters and requires high technical knowledge in photogrammetry. Another important challenge in the RHEA project was thus to automatize the image mosaicking process as much as possible, the objective being to launch and execute the whole processing chain from a single order issued from the RHEA supervising system. In the following, the main steps of the whole processing chain are presented.

\section{General description of the mosaicking process}

\section{Mosaicking principle}

Mosaicking consists of merging several overlapping images into one unique larger image covering the whole scene under study. For this purpose, source images have to be ortho-rectified, i.e. spatially transformed so that they appear as images taken from a zenithal camera with an infinite focal length. If the scene was perfectly flat (assuming no camera lens distortion), ortho-rectification would be a simple geometrical transformation. However, in usual conditions, ortho-rectification requires knowledge of the scene relief, and thus a kind of stereo reconstruction. Therefore, mosaicking of aerial images is made up of several steps:

- First, sets of matching points between various source images are searched for using a feature point extraction algorithm such as the Scale Invariant Feature Transform or SIFT (Lowe, 1999). The 3D positions (x,y,z) of matching points are then determined as well as the 3D position and orientation of the camera for each image acquisition, using a global optimization technique. This results in a "sparse cloud" model, as illustrated in Figure 1a.

- Second, the 3D position of any point in the scene has to be recovered. Because the camera positions are known, the point matching can be made by looking for maximal local correlation between images along epipolar lines. This second step results in a digital surface model (DSM) of the scene (Figure 1b). It requires that 
every point in the scene is viewed at least in two source images, and thus an overlapping of at least $50 \%$ in one direction during image acquisition.

- Once the DSM is computed, the grey level of each 3D point in the scene can be retrieved in the relevant source images, and the ortho-rectified images can be built and merged.

These various steps make up the bulk of the Micmac software suite.

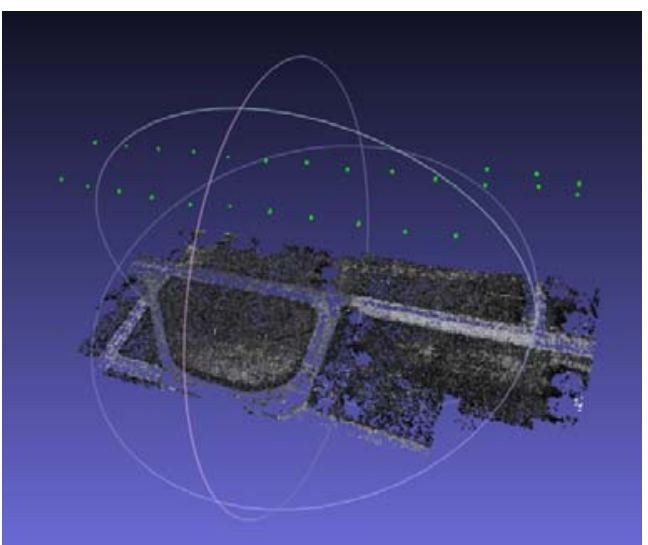

a

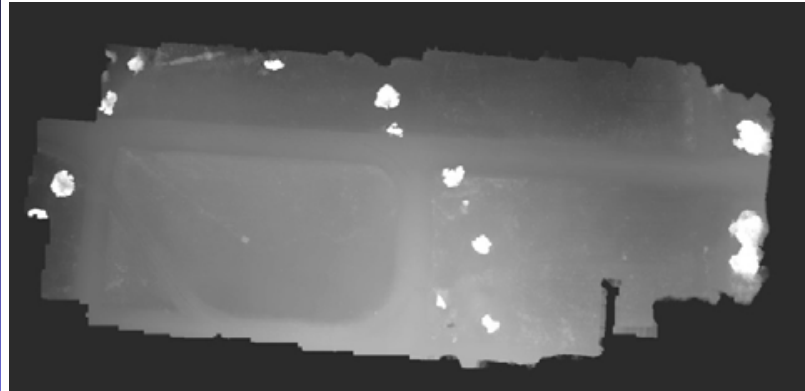

b

Figure1: DSM reconstruction during mosaicking process. a) sparse cloud. b) DSM. Green points in a) correspond to camera positions. White spots in b) correspond to trees.

\section{Geo-referencing}

The sparse cloud and DSM described above are first expressed in relative coordinates, the length unit being the pixel camera size. In order to re-scale them according to the real world coordinates, a set of ground control points (GCP) must be available (or precise IMU and GPS data, which are unavailable on small UAV hardware). It consists in some particular points in the scene, for which the 3D coordinates are known as well as the pixel coordinates in relevant images. Usually, GCP are made of artificial targets or natural points (cross-roads, trees, etc.) whose position has been previously determined, and that are visually retrieved in the source images. In the RHEA project, artificial color targets have been settled around the field and automatically retrieved by image processing, as explained in the next section.

\section{Multispectral management}

Basically, the mosaicking process must be applied on grey level source images. In the usual case, source images are color images captured from a single camera, and thus a luminance image is computed as a combination of the RGB channels for mosaicking computations (feature point and correlation matching). The color information is recovered at the last step, i.e. during source image ortho-rectification and merging.

In the RHEA case, images are captured from two cameras, for which acquisition positions are slightly different. Therefore, two management possibilities can be considered: 
- a) Two different color and NIR mosaic images are built separately, and then superimposed based on their geo-referencing. This solution just requires detecting automatically the GCP in both kinds of images.

- b) Original images from the two cameras are registered at each acquisition. A single mosaicking process is then launched using the color images, and NIR information is recovered at the last step of mosaic image building.

The second solution appears much more complicated, mainly because it requires an accurate registration between different kinds of images (color and NIR), which is not straight forward (see next section). Nevertheless, this solution has been preferred, because it preserves the spectral content of the mosaic image to be affected by mosaicking accuracy (i.e. by positioning differences between color and NIR bands).

\section{Mosaicking process automatization}

\section{Image acquisition devices}

The image acquisition system has been built around two compact still cameras DP2 Merrill (Sigma, Kawasaki, Japan), embedded on a UAV AR 200 (AirRobot ${ }^{\circledR ~ G m b H, ~}$ Arnsberg, Germany). These are high-end commercial cameras, with a 3 layer Foveon ${ }^{\circledR}$ CCD sensor of $4704 \times 3136$ pixels and $30 \mathrm{~mm}$ focal length. One of the cameras was modified for NIR acquisition (internal NIR blocking filter removed and replaced by an external NIR band pass filter). Both cameras have also been modified to allow image shooting through an external signal provided by the UAV control unit (Figure 2).

With a flight altitude of $60 \mathrm{~m}$, images cover a ground area of about $40 \mathrm{x} 30 \mathrm{~m}$, with $1 \mathrm{~cm}$ per pixel. Before each aerial mission, a flight program specifying UAV position for every image acquisition is established accordingly, so that the crop parcel is entirely covered with an image overlapping of $60 \%$ in both directions.

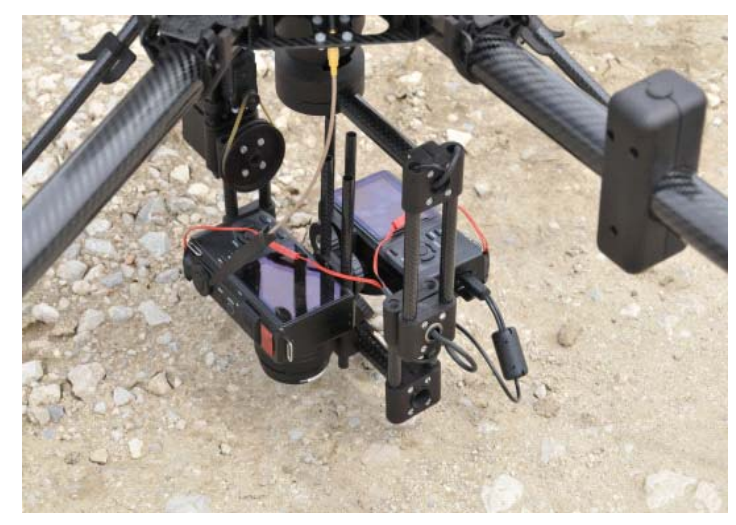

Figure 2. Two Sigma DP2 Merril cameras mounted on UAV

\section{Camera picture synchronization and prepositioning}

During every aerial mission, a flight log file containing actual UAV time-stamped track points and heading issued from an embedded GPS receiver and compass is recorded. 
After the flight, a software module compares this log file with the time data included in Exif metadata of each image (Exchangeable image file format), to recover its position and orientation. This allows roughly prepositioning each image in a local geographic system for further use (Figure 3).

\section{GCP automatic detection and labelling}

Specific targets were designed for automatic detection as GCP. They consist of $30 \mathrm{~cm}$ wood disks with two colors: blue for easy recognition in crop field environment and grey for lighting correction purpose (not used yet). These targets were roughly distributed around the field before the aerial mission, and their 3D position collected using a Real Time Kinematic (RTK) GPS receiver (e.g. Leica RTK GPS, Leica Geosystems ${ }^{\circledR}$, Heerbrugg, Switzerland). Typically 8 to 10 targets are required, and a specific number label is associated to each one during RTK measurement.

At the beginning of the mosaicking process, GCP are systematically searched in every color image and their pixel coordinates stored. The detection process starts with an image binarization using a color look up table to select blue pixels. Resulting binary objects are then checked according to minimal and maximal area values. Finally, an elliptical model is fitted to their external contour (Fitzgibbon et al, 1999). Objects with a ratio between major and minor ellipse radius lower than a given threshold (typically 1.2) are retained, and the ellipse center is stored in floating point pixel coordinates.

Once all targets in all images have been detected, their pixel position is reported in the image prepositioning representation (Figure 3), as well as RTK positioning data. This allows recovering their number label: the label of the nearest RTK position data is chosen, provided the second nearest is at least twice the distance (otherwise the detected target is declared ambiguous and not retained).

\section{$\underline{\text { Registration of color and NIR images }}$}

Due to the very different nature of NIR and color images in a vegetation context, a registration approach based on the Fourier-Mellin transform has been developed. First, images are partitioned in small sub-images on which translation, rotation and scaling between color and NIR bands are identified using Fourier spectrum analysis. Finally, a global homographic transformation between both images is determined. Radial distortion of both cameras is taken into account as well. More details can be found in (Rabatel \& Labbé, 2014). Fourier computations were implemented on the computer Graphics Processing Unit (GPU). An example of image registration is given in Figure 4.

\section{Global processing chain summary}

Figure 5 summarizes the flow chart of the global processing chain. Inputs consist of two text files and both camera SD card contents. Internal software modules have been implemented as independent command line executables managed by a supervisor module, as well as MicMac command line executables. Some complementary modules (such as Sigma raw image conversion in TIFF format) are not represented. 


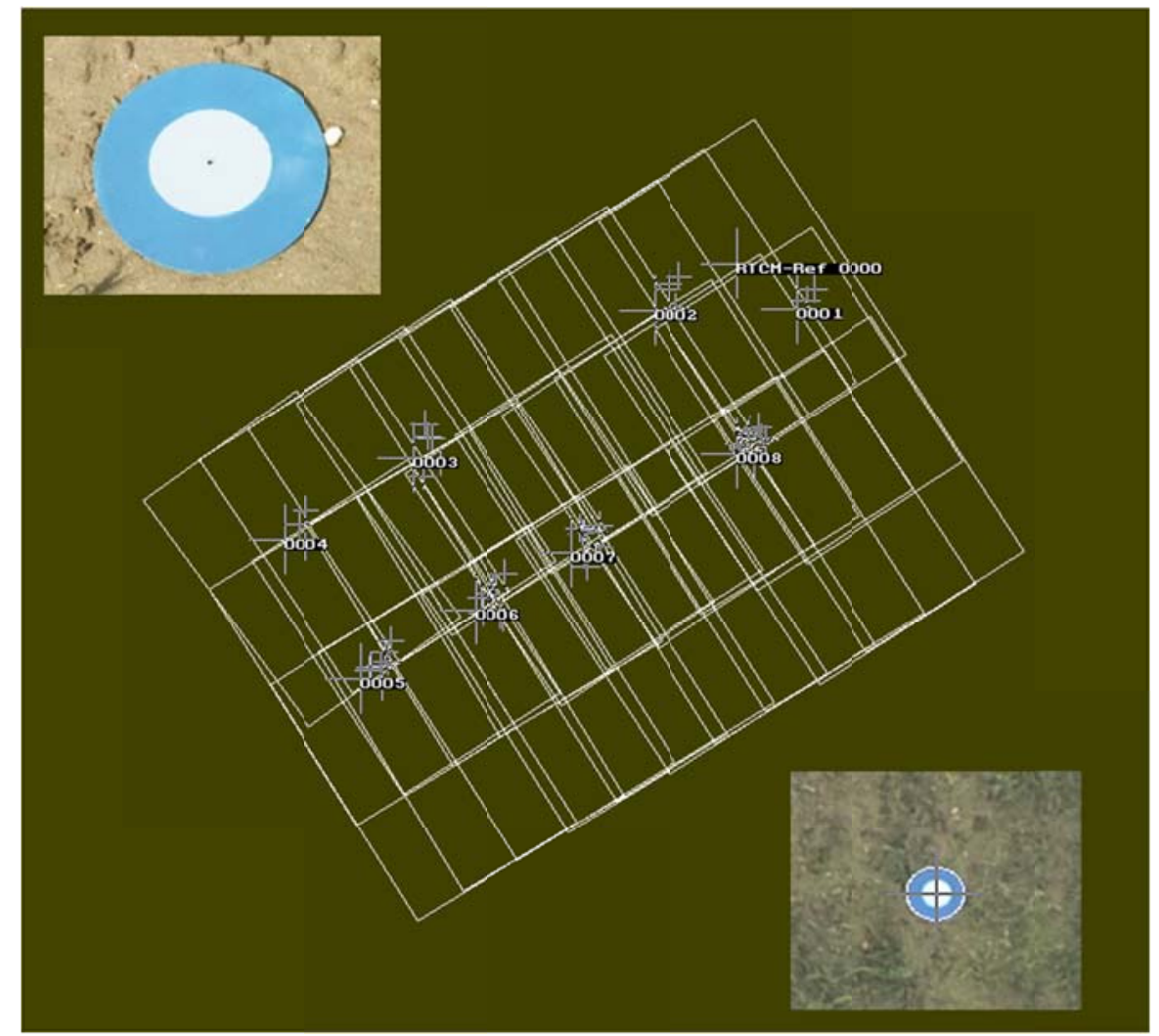

Figure 3. Automatic target detection and labelling

Upper left: detail of a color target; lower right: target_detection; center: prepositioning and target labelling of source images
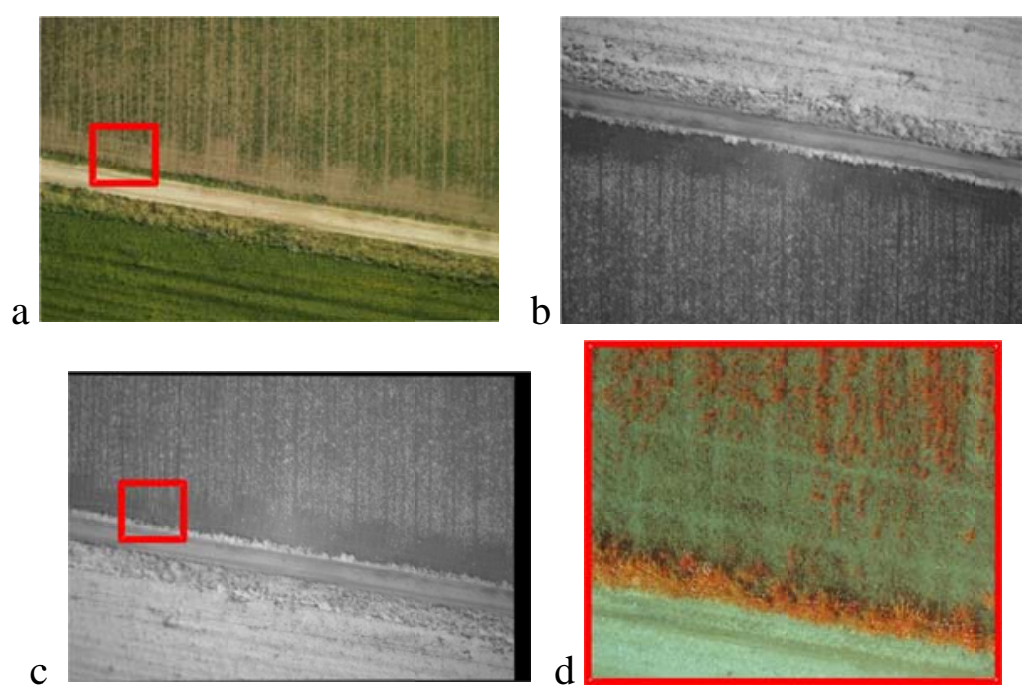

Figure 4. Registration of color and NIR images.

a) original color image; b) original NIR image; c) straightened NIR image

d) Detail of resulting 4-channel image $a+c$ (false color representation) 


\section{Discussion and conclusion}

This processing chain has been successfully applied to various aerial missions on wheat and maize crops in the context of the RHEA project, in December 2013 (Montpellier area, France, $43.6469^{\circ} \mathrm{N}, 3.8827^{\circ} \mathrm{E}$ ), January and April 2014 (Arganda, Spain, $40.3160^{\circ} \mathrm{N}, 3.4865^{\circ} \mathrm{W}$ ). Target detection and color-NIR registration had proven to be particularly robust. Though variable translations between 100 and to 300 pixels were observed between color and NIR images, mainly due to random shooting latency of the cameras, residual registration error remained below 0.5 pixels in every case.

As an example, for a set of 39 images covering approximately 1 ha (120mx85 m), total processing time was about 3h on a PC computer equipped with a 4-core i7-4800MQ processor and a NVIDIA Quadro L610M GPU (2.5 hours for MicMac mosaicking process, $30 \mathrm{~min}$ for image TIFF conversion and color-NIR registration).

As a conclusion, this automatized processing chain could bring a significant contribution to the promotion of UAV aerial imagery in various precision agriculture applications, by facilitating the acquisition process. The only remaining manual operations concern the GCP distribution and position measurement in the field (which could be avoided by using fixed targets in some cases), and the SD card transfer from the cameras to the computer. Next improvements concern the management of image acquisition with automatic exposure (which requires tuning image conversion gain according to Exif data) and reflectance correction using GCP as reference to improve the radiometric quality of the resulting mosaic image.

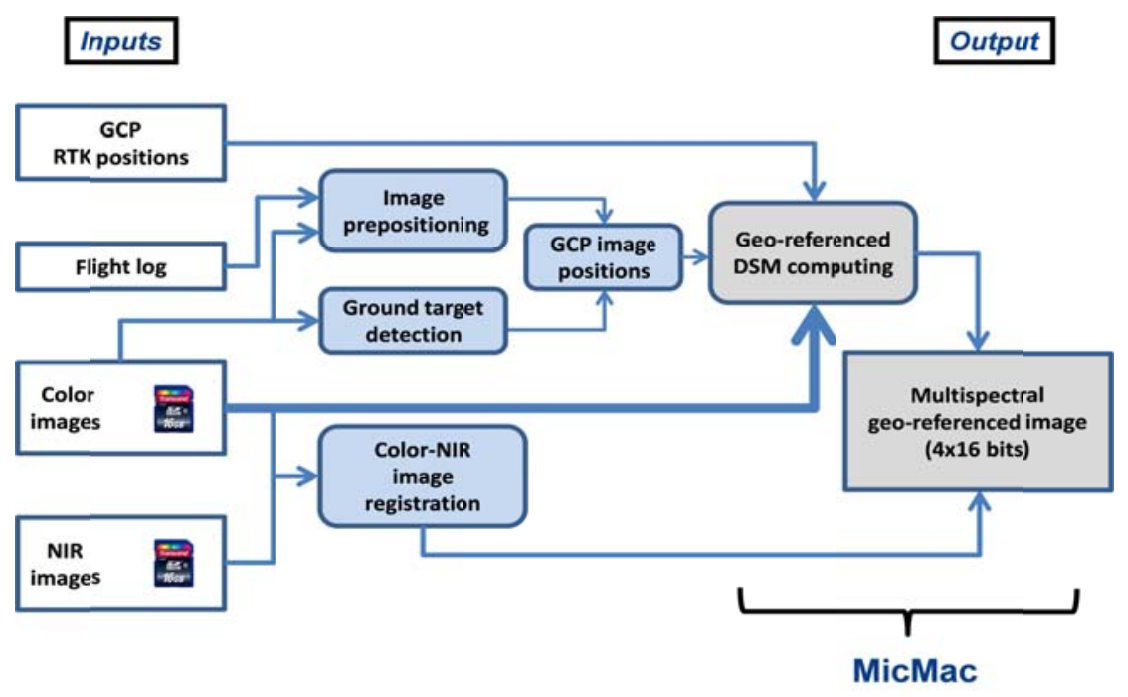

Figure 5: Global processing flow chart.

\section{Acknowledgements}

The research leading to these results has received funding from the European Union's Seventh Framework Program [FP7/2007-2013] under grant agreement n²45986. 


\section{References}

- Fitzgibbon, A., Pilu, M., Fisher, R. B., 1999. Direct Least Square Fitting of Ellipses, IEEE Transactions on Pattern Analysis and Machine Intelligence 21, pp 476-480 (1999)

- García-Ruiz, F., Sankaran, S., Maja, J.M., Lee, W.S., Rasmussen, J., Ehsani, R., 2013. Comparison of two aerial imaging platforms for identification of Huanglongbing-infected citrus trees. Computers and Electronics in Agriculture 91, 106-115 (2013)

- Gée C., Bossu J., Jones G., Truchetet F., 2008. Crop/Weed discrimination in perspective agronomic images. Computers and Electronics in Agriculture, 60/1, 49-59.

- Lowe, D.G., 1999. Object recognition from local scale-invariant features. In: Proc. of the International Conference on Computer Vision, Corfu, Greece, pp 1150-1157

- Micmac, IGN. http://logiciels.ign.fr/?-Micmac,3. Last accessed 02-26-2015

- Shahbazi, M., Théau J., Ménard P., 2014. Recent applications of unmanned aerial imagery in natural resource management, GIScience \& Remote Sensing, 51(4), 339-365

- Peña, J.M., Torres-Sánchez, J., de Castro, A. I., Serrano-Pérez, A., LópezGranados, F., 2014. Comparing visible and color-infrared UAV imagery for early-season weed mapping: the case of maize as a wide row crop. In: RHEA 2014, 2nd International Conference on Robotics and associated Hightechnologies and Equipment for Agriculture and forestry, Madrid, Spain, pp 319-328, http://www.rhea-project.eu

- Rabatel, G, Labbé, S. - 2014. Registration of visible and near infrared aerial images based on Fourier-Mellin Transform. In: RHEA 2014, 2nde International Conference on Robotics and associated High-technologies and Equipment for Agriculture and forestry, Madrid, Spain, 329-338. http://www.rhea-project.eu

- Slaughter D.C., Giles D.K., Downey D., 2008. Autonomous robotic weed control systems: A review. Computers and Electronics in Agriculture, 61, 63-78

- Torres-Sánchez J., López-Granados F., de Castro A.I., Peña. J.M., 2014. Multitemporal weed mapping using UAV imagery for early site-specific control: the case of wheat as a narrow row crop. In: RHEA 2014, 2nd International Conference on Robotics and associated High-technologies and Equipment for Agriculture and forestry, Madrid, Spain, 269-278. http://www.rhea-project.eu

- Xiang, H., Tian, L., 2011. Development of a low-cost agricultural remote sensing system based on an autonomous unmanned aerial vehicle (UAV). Biosystems Engineering, 108( 2), 174-190.

- Zhang, C., Kovacs J.M., 2012. The Application of Small Unmanned Aerial Systems for Precision Agriculture: A Review. Precision Agriculture 13(6) 693712. 